\title{
Front Matter: Volume 11276
}

, "Front Matter: Volume 11276," Proc. SPIE 11276, Optical Components and Materials XVII, 1127601 (7 April 2020); doi: 10.1117/12.2567553

SPIE. Event: SPIE OPTO, 2020, San Francisco, California, United States 


\section{PROCEEDINGS OF SPIE}

\section{Optical Components and Materials XVII}

Shibin Jiang

Michel J. F. Digonnet

Editors

4-6 February 2020

San Francisco, California, United States

Sponsored and Published by

SPIE 
The papers in this volume were part of the technical conference cited on the cover and title page. Papers were selected and subject to review by the editors and conference program committee. Some conference presentations may not be available for publication. Additional papers and presentation recordings may be available online in the SPIE Digital Library at SPIEDigitalLibrary.org.

The papers reflect the work and thoughts of the authors and are published herein as submitted. The publisher is not responsible for the validity of the information or for any outcomes resulting from reliance thereon.

Please use the following format to cite material from these proceedings:

Author(s), "Title of Paper," in Optical Components and Materials XVII, edited by Shibin Jiang, Michel J. F. Digonnet, Proceedings of SPIE Vol. 11276 (SPIE, Bellingham, WA, 2020) Seven-digit Article CID Number.

ISSN: 0277-786X

ISSN: 1996-756X (electronic)

ISBN: 9781510633155

ISBN: 9781510633162 (electronic)

Published by

SPIE

P.O. Box 10, Bellingham, Washington 98227-0010 USA

Telephone +1 3606763290 (Pacific Time) · Fax +1 3606471445

SPIE.org

Copyright (C) 2020, Society of Photo-Optical Instrumentation Engineers.

Copying of material in this book for internal or personal use, or for the internal or personal use of specific clients, beyond the fair use provisions granted by the U.S. Copyright Law is authorized by SPIE subject to payment of copying fees. The Transactional Reporting Service base fee for this volume is $\$ 18.00$ per article (or portion thereof), which should be paid directly to the Copyright Clearance Center (CCC), 222 Rosewood Drive, Danvers, MA 01923. Payment may also be made electronically through CCC Online at copyright.com. Other copying for republication, resale, advertising or promotion, or any form of systematic or multiple reproduction of any material in this book is prohibited except with permission in writing from the publisher. The CCC fee code is 0277$786 \mathrm{X} / 20 / \$ 18.00$.

Printed in the United States of America.

Publication of record for individual papers is online in the SPIE Digital Library.

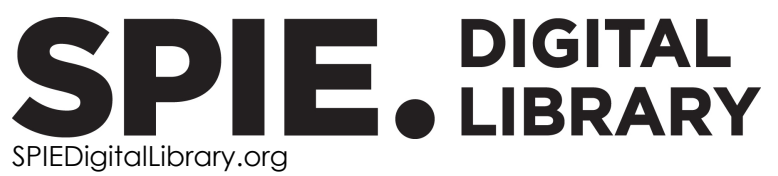

Paper Numbering: Proceedings of SPIE follow an e-First publication model. A unique citation identifier (CID) number is assigned to each article at the time of publication. Utilization of CIDs allows articles to be fully citable as soon as they are published online, and connects the same identifier to all online and print versions of the publication. SPIE uses a seven-digit CID article numbering system structured as follows:

- The first five digits correspond to the SPIE volume number.

- The last two digits indicate publication order within the volume using a Base 36 numbering

system employing both numerals and letters. These two-number sets start with 00, 01, 02, 03, 04,

05, 06, 07, 08, 09, 0A, OB ... 0Z, followed by 10-1Z, 20-2Z, etc. The CID Number appears on each

page of the manuscript. 


\section{Contents}

$\begin{array}{ll}\text { vii } & \text { Authors } \\ \text { ix } & \text { Conference Committee }\end{array}$

OPTICAL SWITCHES

1127602 Phase change material integrated silicon photonics: GST and beyond (Invited Paper) [1 1276-1]

1127603 Integrated electroholographic photorefractive gratings with sustained longevity by LED illumination [1 1276-2]

1127604 Figure of merit of phase change materials for Mach-Zehnder interferometer-based and directional-coupler-based optical switches [1 1276-3]

\section{OPTICAL MODULATORS}

1127607 Free-spectral-range-free microring-based coupling modulator with integrated contradirectional-couplers [11276-6]

1127608 Composite material hollow core anti-resonant fiber electromodulators: exploring the optical FET response [11276-7]

1127609 Phase-locked fiber interferometer with high frequency, low voltage fiber-stretcher and application to optical field reconstruction [1 1276-8]

\section{PHOTODETECTORS}

11276 OG High-sensitivity NIR photodiodes using black silicon [1 1276-15]

$11276 \mathrm{OH} \quad$ Dilute nitride photodetector arrays for sensing applications [1 1276-16]

11276 Ol Broadband PureGaB Ge-on-Si photodiodes responsive in the ultraviolet to near-infrared range [11276-17]

11276 OJ Characterization of HOT MWIR InAs/InAsSb T2SL discrete photodetectors [1 1276-60] 


\section{RARE-EARTH-DOPED LASERS}

11276 OK Modeling and parameter recovering of rare-earth-doped/co-doped glass and glass ceramics optical devices (Invited Paper) [1 1276-18]

$11276 \mathrm{OL} \quad$ Low numerical aperature large-mode-area neodymium-doped fibers fabricated by SPCVD and ASD for laser operation near 920nm [1 1276-19]

11276 OM Double line neodymium doped $\mathrm{GeO}_{2}-\mathrm{PbO}$ waveguide amplifier for the second telecom window [1 1276-20]

$11276 \mathrm{ON} \quad$ Polydispersed $\mathrm{Nd}^{3+}: \mathrm{YVO}_{4}+\mathrm{SiO}_{2}$ powders for highly efficient random lasers [1 1276-21]

\section{OPTICAL PROPERTIES OF MATERIALS}

11276 OP Graphene material characterisation for optofluidic applications [1 1276-23]

11276 OR Breaking the silica ceiling: ZBLAN-based opportunities for photonics applications [1 1276-25]

\section{LASERS AND AMPLIFIERS}

11276 OS Reconsidering nanoparticles in optical fibers (Invited Paper) [11276-26]

$11276 \mathrm{OV}$ Continuous linewidth funing of a laser source from single frequency to over $30 \mathrm{GHz}$ using phase modulation amplification using cascaded four-wave mixing [1 1276-29]

11276 OW Hundreds of meter-long low-loss silicon-core optical fiber [11276-30]

\section{SENSORS}

11276 OY High-temperature enhanced Rayleigh scattering optical fiber sensor for borehole applications [11276-32]

$112760 Z$ High sensitivity refractive index sensing using an axicon lens structure [11276-33]

1127611 Enhanced modal interference in negative curvature fiber for sensing applications [1 1276-35]

1127612 Synthetic diamond lenses for multi-spectral imaging [11276-36]

$1127613 \quad$ OCT measurement of aspheric polymer lenses for adaptive assembly of micro optical imaging objectives [11276-37] 
$1127614 \quad$ Photonic glass ceramics based on $\mathrm{SnO}_{2}$ nanocrystals: advances and perspectives (Invited Paper) [11276-38]

\section{NANOSTRUCTURES}

1127619 Optical scattering measurements of random anti-reflection subwavelength surface structures on binary gratings [11276-44]

\section{POSTER SESSION}

$112761 \mathrm{~A}$ Investigation of phosphate glasses for white-light emission [11276-45]

11276 1B Parity-time symmetry laser with nanocolloidal gain medium [1 1276-46]

11276 1D Materials development and mid-infrared emission properties of $\mathrm{Dy}$-doped $\mathrm{TIPb}_{2} \mathrm{Br}_{5}$ and $\mathrm{CsPbCl}_{3}$ [11276-48]

$112761 \mathrm{E} \quad$ Characterization of a Sagnac loop mirror-based hybrid passive variable optical coupler/attenuator [11276-49]

$11276 \mathrm{IF} \quad$ Fabrication of a tellurite hollow core optical fiber for mid-infrared transmission [1 1276-50]

$1127611 \quad$ Compact passive photonic filter using an MZI [1 1276-53]

$112761 \mathrm{~K} \quad$ Optimally designed narrowband guided-mode resonance transmittance filters for label-free optical biosensor [11276-55]

11276 IL Custom high-sensitivity CCD and SCMOS detectors for high-harmonic generation, $x$-ray absorption spectroscopy, soft $\mathrm{x}$-ray microscopy/tomography, and hard $\mathrm{x}$-ray detection [11276-56]

1127610 Persistent luminescence features in hexagonal Sro.75 $\mathrm{Al}_{1.5} \mathrm{Si}_{0.5 \mathrm{O}_{4}: \mathrm{Eu}^{2+}}$ phosphor [1 1276-59] 
Proc. of SPIE Vol. 11276 1127601-6

\section{Downloaded From: https://www.spiedigitallibrary.org/conference-proceedings-of-spie on 26 Apr 2023
Terms of Use: https://www.spiedigitallibrary.org/terms-of-use}




\section{Authors}

Numbers in the index correspond to the last two digits of the seven-digit citation identifier (CID) article numbering system used in Proceedings of SPIE. The first five digits reflect the volume number. Base 36 numbering is employed for the last two digits and indicates the order of articles within the volume. Numbers start with 00, 01, 02, 03, 04, 05, 06, 07, 08, 09, OA, OB...0Z, followed by 10-1Z, 20-2Z, etc.

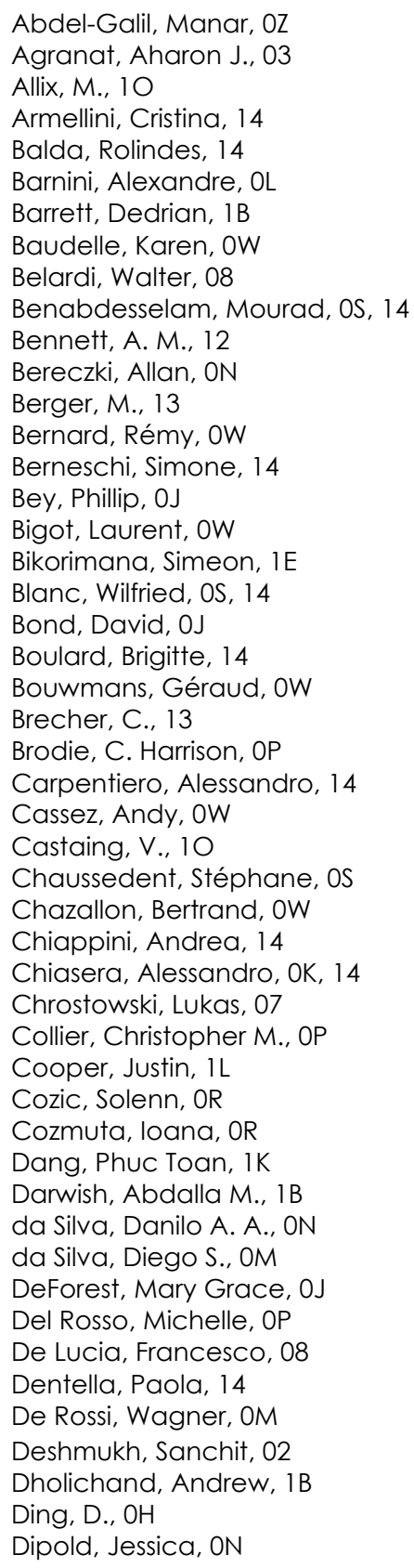

Dorosz, Dominik, 14

Dorsinville, Roger, $1 \mathrm{E}$

Dowd, P., OH

Eaton, Shane, 14

El Hamzaoui, Hicham, OW

Falconi, Mario Christian, OK, 14

Fang, Zhuoran, 02

Faulkner, F. R., 12

Feder, Kenneth S., OY

Fernandez, Joaquin, 14

Fernández-Carrión, A. J., 10

Ferrari, Maurizio, OK, 14

Fourmont, Jorel, OS

Frishman, Sagi, 03

Gadamsetti, Praneeth, 19

Gao, L., $\mathrm{OH}$

Garcia, Yehudit, 03

Gates, James, 14

Girard, Sylvain, OL

Gluchowski, Pawel, OK, 14

Goel, Charu, 11

Goodson, Mersaydes, IA

Guillermier, Christelle, OS

Guitton, Pascal, OL

Haarahiltunen, Antti, OG

Habert, Rémi, OW

Hammood, Mustafa, 07

Hart, D., ID

Hayes, John R., 08

Heinonen, Juha, OG

Hewak, Dan, 08

Hoeren, M., 13

Hömmerich, U., ID

Hossain, Abdullah, 1E

Huang, Chung-Che, 08

Hussain, A., 11

Ischia, Gloria, 14

Ismail, Yehea, $0 Z$

Jaeger, Nicolas A. F., 07

Jimenez-Villar, E., ON

Juntunen, Mikko A., OG

Kabir, A., 1D

Kassab, Luciana R. P., OM

Katzenmeyer, Aaron M., 09

Kervella, Louanne, OL

Khalii, Diaa, $0 Z$

Kim, Jongwoo, $0 \mathrm{~J}$

Kimchi, Joe, 0J

Knežević, Tihomir, 0 I 
König, N., 13

Krakers, Max, 이

Kudinova, Maryna, OW

Kunala, Karteek, 19

Kuwahara, Masashi, 04

Laneve, Dario, OK

Laroche, Mathieu, OL

Le Corre, Kilian, OL

Lee, Ji-Hoon, $1 \mathrm{~K}$

Lewis, Adam H., 08

Lewis, Kaiana, 1B

Lin, Richard, $1 \mathrm{E}$

Lin, Stephen, 07

Lu, Zhuorvi, OS

Lukowiak, Anna, OK, 14

Mady, Franck, OS, 14

Majumdar, Arka, 02

Malik, F., 11

Maros, A., $\mathrm{OH}$

Martin, Isabelle, OS

Martini, Jose R. L., OR

Massella, Damiano, 14

Mistry, Ajay, 07

Molardi, Carlo, OS

Monberg, Eric M., OY

Monteiro, C., 10

Nanver, Lis K., Ol

Narain, Morgan, 1B

Naydenkov, M., $\mathrm{OH}$

Nishiharaguchi, Nobuhiko, IF

Nunzi Conti, Gualtiero, 14

Ohishi, Yasutake, IF

Parkins, Sharon, 1B

Pasanen, Toni P., OG

Patel, Darayas N., 1B

Piarresteguy, Andrea, OS

Pigeonneau, Franck, OS

Plus, Stéphane, OW

Poletti, Francesco, 08

Pop, Eric, 02

Poulain, Marcel, OR

Poulain, Samuel, $O R$

Poutous, Menelaos K., 19

Prakash, Roopa, OV

Prudenzano, Francesco, OK, 14

Qadir, M. F., 11

Ramponi, Roberta, 14

Reddy, B. Rami, 1A

Riediger, M., 13

Righini, Giancarlo C., 14

Robin, Thierry, OL

Rosenthal, Daniel, 03

Rossi, Barbara, 14

Roucka, R., OH

Rumyantsev, Andrey, 0J

Saint-Cyr, Hugues François, OS

Sakamoto, Kenta, 04

Sarkisov, Sergey S., 1B

Savin, Hele, OG

Sazio, Pier-John, 08, 14
Schmitt, R., 13

Serve, Michael Dov, OG

Shenk, Scott D., OY

Shoman, Hossam, 07

Siala, S., $\mathrm{OH}$

Simoff, Debra A., OY Speranza, Giorgio, 14

Stolov, Andrei A., OY

Suarez, F., $\mathrm{OH}$

Supradeepa, V. R., OV

Suzuki, Takenobu, $1 \mathrm{~F}$

Swillam, Mohamed, $0 Z$

Tahir, W., 11

Tong, Hoang Tuan, IF

Tosi, Daniele, OS

Tran, Lam Thi Ngoc, OK, 14

Trivedi, S. B., ID

Troles, Johann, OW

Tsuda, Hiroyuki, 04

Twitchen, D. J., 12

Ummy, Muhammad A., $1 \mathrm{E}$

Vähänissi, Ville, OG

Vanvincq, Olivier, OW

Varagnat, Antoine, $1 \mathrm{~L}$

Varas, Stefano, 14

Vermillac, Manuel, OS

Viana, B., 10

Vikram, B. S., OV

Walker, Sean, 1B

Werner, Lutz, OG

Wetter, Niklaus U., OM, ON

Wise, Adam J., $1 \mathrm{~L}$

Woodward, Thomas, $1 \mathrm{~L}$

Wu, Hongchao, OY

$\mathrm{XU}$, Peipeng, 02

Yang, C., ID

Yoo, Seongwoo, 11

Yuan, Henry, 0J

Zakwan, M., 11

Zang, Jichao, 11

Zheng, Jiajiu, 02

Zonta, Daniele, 14

Zontar, D., 13

Zur, Lidia, OK, 14 


\section{Conference Committee}

Symposium Chairs

Sailing He, KTH Royal Institute of Technology (Sweden) and Zhejiang University (China)

Yasuhiro Koike, Keio University (Japan)

Symposium Co-chairs

Connie J. Chang-Hasnain, University of California, Berkeley (United States)

Graham T. Reed, Optoelectronics Research Center, University of Southampton (United Kingdom)

Program Track Chairs

James G. Grote, Photonics Engineering Consultant (United States)

Shibin Jiang, AdValue Photonics, Inc. (United States)

Conference Chairs

Shibin Jiang, AdValue Photonics, Inc. (United States)

Michel J. F. Digonnet, Stanford University (United States)

Conference Program Committee

Jean-Luc Adam, Université de Rennes 1 (France) Joel Bagwell, Edmund Optics Inc. (United States)

Rolindes Balda, Universidad del País Vasco (Spain)

Robert P. Dahlgren, NASA Ames Research Center (United States)

Angel Flores, Air Force Research Laboratory (United States)

Jesse A. Frantz, U.S. Naval Research Laboratory (United States)

Leonid B. Glebov, CREOL, The College of Optics and Photonics, University of Central Florida (United States)

Seppo K. Honkanen, University of Eastern Finland (Finland) and Microsoft HoloLens (Finland)

Jacques Lucas, Université de Rennes 1 (France)

Yasutake Ohishi, Toyota Technological Institute (Japan)

Aydogan Ozcan, University of California, Los Angeles (United States)

Giancarlo C. Righini, Istituto di Fisica Applicata "Nello Carrara" (Italy)

Setsuhisa Tanabe, Kyoto University (Japan)

John M. Zavada, Polytechnic Institute of New York University (United States)

Jun Zhang, U.S. Army Research Laboratory (United States) 


\section{Session Chairs}

1 Optical Switches

Shibin Jiang, AdValue Photonics, Inc. (United States)

2 Optical Modulators

John M. Zavada, Polytechnic Institute of New York University (United States)

3 Plasmonic Devices and Technologies

Michel J. F. Digonnet, Stanford University (United States)

4 Photodetectors

Seppo K. Honkanen, University of Eastern Finland (Finland)

5 Rare-Earth-Doped Lasers

Rolindes Balda, Universidad del País Vasco (Spain)

6 Optical Properties of Materials

Michel J. F. Digonnet, Stanford University (United States)

$7 \quad$ Lasers and Amplifiers

Jesse A. Frantz, U.S. Naval Research Laboratory (United States)

8 Sensors

Shibin Jiang, AdValue Photonics, Inc. (United States)

9 Nanoparticles

Michel J. F. Digonnet, Stanford University (United States)

10 Nanostructures

Michel J. F. Digonnet, Stanford University (United States) 